\title{
Transformation and Optimization of Rural Ecological Endowment Industry Chain Based on Constrained Clustering Algorithm
}

\author{
Yan Zhao $\mathbb{D}^{1,2,3}$ and Zhiyi Gai ${ }^{1}$ \\ ${ }^{1}$ School of Economics and Management, Inner Mongolia Agricultural University, Hohhot 010019, China \\ ${ }^{2}$ School of Economics and Management, Inner Mongolia Normal University, Hohhot 010022, China \\ ${ }^{3}$ Hainan University, Haikou 570228, China \\ Correspondence should be addressed to Yan Zhao; zy_19980901@163.com
}

Received 14 November 2021; Revised 15 December 2021; Accepted 16 December 2021; Published 24 January 2022

Academic Editor: Tongguang Ni

Copyright (c) 2022 Yan Zhao and Zhiyi Gai. This is an open access article distributed under the Creative Commons Attribution License, which permits unrestricted use, distribution, and reproduction in any medium, provided the original work is properly cited.

\begin{abstract}
China is experiencing a serious aging crisis, with the number of elderly people rising at an unprecedented rate. First and foremost, the pension industry's service object is the pension. To build an old-age industry complex, make use of the unique natural resources in the countryside, provide the original ecological old-age care services, and transform, optimize, and make efficient use of the existing policies and technologies. The drawbacks of the traditional pension model are becoming increasingly apparent. We must investigate a new pension model in order to ensure the long-term development of China's pension system and reduce the increased pension burden on governments at all levels. The rural areas' unique natural resources should be utilized to provide unique ecological elderly care services, and transformation and optimization must be implemented (that is, making efficient use of existing policies and technology to construct an elderly care industry complex). As a new pension mode for the elderly to become more connected to the natural world and improve their physical and mental health, ecological pensions have emerged as a new economic growth point that can fill the gap in the pension industry's supply while also meeting the elderly's growing spiritual and cultural needs. A constrained clustering algorithm is proposed in this article. Unsupervised learning is used in the constrained clustering algorithm. The clustering algorithm must determine the data objects to be clustered because they are not labeled. Because the data objects have no prior knowledge, the clustering algorithm analyzes them using the same principles. The effectiveness of the clustering results is determined by the dataset's adherence to the previously stated principles. The constrained clustering algorithm has greatly improved the transformation and optimization of the rural ecological elderly care industrial chain.
\end{abstract}

\section{Introduction}

With the deepening of China's aging population, the pension industry has gradually become a new sunrise industry, with an immeasurable development prospect [1]. China has been involved in a serious wave of aging, and the number of elderly people is increasing at an unprecedented rate. The existing old-age care institutions are far below the actual expectations of the people in terms of number and service quality [2]. The number of people over 60 years old will increase from 178 million to 221 million, with an average annual increase of 8.6 million. The proportion of the elderly population will increase from $13.3 \%$ to $16 \%$, with an average annual increase of 0.54 percentage points. According to the forecast of the National Bureau of Population Statistics, by 2050, China's elderly population will reach 437 million, accounting for $30 \%$ of the total population, and the number will exceed the sum of the elderly population of developed countries in the world [3]. China is about to become a highly aging society, and the problem of providing for the aged has seriously restricted the development of China's economy. From the whole development process and coverage of the old-age care industry, it can be shown that the main characteristics of the old-age care industry are wide coverage, long industrial chain, sustainability, and environmental friendliness. First of all, the service object of the 
pension industry is pension. We made use of the unique natural resources in the countryside, provided the original ecological old-age care services, and transformed, optimized, and made efficient use of the existing policies and technologies to build an old-age industry complex [4]. The disadvantages of the traditional old-age care model are becoming more and more obvious. In order to make the long-term development of China's old-age care reduce the burdens of the old-age care on the governments at all levels, which are getting heavier and heavier, we must explore a new old-age care model [5]. Old-age care mainly includes family care, community care, and institutional care. Institutional care in real life includes pension yuan, nursing homes, and apartments for the elderly. These institutions are either public institutions or private institutions that provide oldage care services. As a new old-age care model, ecological old-age care, which enables the elderly to get close to the ecological environment and gain physical and mental health, has become a new economic growth point. Comparatively speaking, the investment risk of social capital is relatively high, and a complete mature model of investment in old-age care institutions has not yet been formed and most of the capital is still on the sidelines, which make it difficult for the government to really implement measures to encourage social forces to enter the old-age care industry $[6,7]$.

The clustering method is an unsupervised learning method. The data objects to be clustered are unmarked and need to be determined by the clustering algorithm itself. Because there is no background knowledge about data objects, the clustering algorithm uses the same principles to analyze these data, and whether the clustering results are effective depends on the degree to which the dataset conforms to the preestablished principles [8]. Clustering is the process of dividing objects into similar object classes according to some attributes of objects [8]. The goal of clustering is to make the objects in the class as similar as possible and the objects between classes as different as possible. Unlike classification [9-11], clustering usually has no prior knowledge or background knowledge as guidance. It is an automatic recognition [12] and unsupervised learning process based on object similarity [13].

The selection of cluster centers is based on the direction relationship instead of random selection, which makes the selection of cluster centers have good distribution and avoids the decrease of convergence speed caused by the concentration of cluster center distribution. Clustering with constraints refers to the method of expressing specific domain knowledge in the form of "constraints" and embedding it into the clustering process $[14,15]$. Because of the use of domain knowledge, the clustering algorithm can obtain more heuristic information, thus reducing the "blindness" in its search process and improving the efficiency and clustering quality. Clustering with constraints refers to the method of expressing specific domain knowledge in the form of constraints and embedding it into the clustering process [16]. Because of the use of domain knowledge, the clustering algorithm can obtain more heuristic information, thus reducing the "blindness" in its search process and improving the efficiency and clustering quality. Supervised learning methods [17-19] require each data record to have a class label, while unsupervised learning methods do not consider any guiding information [20,21]. Because users often have clear application requirements, in many practical applications, effective solutions tend to add user preferences or constraints to the clustering process, which has an important impact on the results of knowledge discovery and helps us find the knowledge patterns that users are interested in or more in line with users' needs. After the constrained clustering algorithm, through the analysis of the decision graph, a series of data points that may be the center of the cluster are selected in various ways, which is called the initial center point. The number of initial center points may be more than the real number of clusters, but the initial center points must be local high-density points in the data. By selecting the initial center point, the potential hierarchical structure information of the system can be extracted, which can serve as a heuristic for subsequent clustering [22].

\section{Related Work}

Literature [23] pointed out that the combination of specialization and integration, the combination of public welfare and marketization, the combination of real estate sales and diversified business, and the combination of independent management and cooperative management should be emphasized in the marketization strategy. In literature [24], through the method of big data analysis, the current old-age policy is not working well at present, the change of family structure has a double influence on old-age care, and the existing old-age care in rural areas is still dominated by families, so the old-age care in rural areas during the social transformation period needs policy support. In literature [25], building and optimizing the pension industry chain is an effective way to promote the healthy and long-term development of China's pension industry, and the key lies in the optimization and integration of the pension industry chain. At present, the definition of industrial chain risk has not reached an agreement in domestic and foreign academic circles. However, for the specific industrial chain, scholars have given a definition of industrial chain risk with strong pertinence but narrow applicability. Literature [26] pointed out that the strategy of healthy China and rural revitalization clearly highlighted the concept of coordinated development of healthy old-age care and rural revitalization in the elaboration of the implementation framework of the two strategies. In literature [27], through the big data analysis method, China has been involved in a serious wave of aging, the number of the elderly population is increasing at an unprecedented rate, and the existing elderly care institutions are far from the actual elderly care service expectations of the people in terms of number and service quality. How to collect the actual needs of the elderly population has become one of the difficulties in implementing such a platform. Literature [28] pointed out that the disadvantages of the traditional pension model are becoming more and more obvious. In order to make the long-term development of China's pension reduce the heavier pension burden of governments at all levels, we must explore a new 
pension model. Literature [28] stated that the construction of leisure agriculture and rural tourism provides better methods for elderly care services. In literature [29], through the big data analysis method, the current willingness of the elderly to choose elderly care institutions for the elderly shows an increasing trend. It is considered that the reasons restricting the economic growth of China's pension institution industrial chain include income level and capital source. The effective way of economic growth of pension institution industrial chain in the future should take the road of industrialization development. Literature [30] showed the agricultural development of the central region, analyzed the main problems and obstacles it faces, advocated the establishment of a reasonable responsibility-sharing mechanism to support the development of agricultural product processing industry, improved rural infrastructure construction, and realized the sustainable and stable development of agriculture. Literature [31] pointed out that for the elderly lifestyle, old customers mostly choose apartments or elderly care centers, followed by hotels and restaurants, which is related to the frugal consumption mentality of the elderly.

\section{Constrained Clustering Algorithm}

3.1. Definition of Constrained Clustering. Constrained clustering refers to a method in which specific domain knowledge is expressed in the form of "constraints" and embedded in the clustering process. $C C D_{\text {default }}$ refers to the updated value, that is, the updated value of enterprise industrial chain tightness calculated regularly through supply chain relevance, enterprise chain relevance, value chain relevance, and spatial chain relevance $\left(\mathrm{Cl}_{1}, \mathrm{Cl}_{2}, \ldots, \mathrm{Cl}_{k}\right)$, so that the objective function DISP $=\sum_{i=1}^{k} \operatorname{disp}\left(C l_{i}\right.$, rep $\left._{i}\right)$ is the smallest, and each class satisfies constraint $c$. Among them, $\operatorname{disp}\left(C l_{i}, \operatorname{rep}_{i}\right)$ is defined as $\sum_{p \in C l i} d f\left(p, \operatorname{rep}_{i}\right)$.

According to the rural ecology, the algorithm formula of correlation degree of pension industry chain is deduced as follows:

$$
\begin{aligned}
\mathrm{OCD}= & \mathrm{CCD}_{\text {default }}+\mathrm{CCD}_{\text {default }}=\mathrm{CCD}_{\text {default }} \\
& +f(\mathrm{SCD}, \mathrm{ECD}, \mathrm{VCD}, \mathrm{RCD}) .
\end{aligned}
$$

$\mathrm{CCD}_{\text {default }}$ refers to the default value, that is, the initial value of the algorithm calculated according to the basic information of the enterprise. $\mathrm{CCD}_{\text {default }}$ refers to the updated value, that is, the updated value of enterprise industrial chain tightness calculated regularly through supply chain relevance, enterprise chain relevance, value chain relevance, and spatial chain relevance. According to the above $C C D_{\text {default }}$ calculation principle, the $\mathrm{CCD}_{\text {default }}$ decomposition formula is derived as follows:

$$
\mathrm{CCD}_{\text {default }}=\sum_{i=1}^{n} B_{i} \times W_{i} \text {, }
$$

where $n$ represents the amount of enterprise basic resource information involved in the calculation; it means the evaluation parameter calculated by the Ith basic resource information of $B_{i}$ according to the set judgment conditions, which should meet $0 \leq B_{i}$, $\leq 1$. It indicates the importance percentage of the Ith basic resource information in the basic information of the enterprise. All the importance percentages must meet the following conditions: $0 \leq W_{i} \leq 1$, $W_{1}+W_{2}+\cdots+W_{n}=1$.

According to the above description of the four dimensions of the industrial chain, $\mathrm{CCD}_{\text {default }}$ is composed of four basic parameter combinations: SCD, ECD, VCD, and RCD. Set the importance percentage of these four basic parameters in $\mathrm{CCD}_{\text {default }}$ as $\alpha, \beta, \gamma, \vartheta$, respectively, and then use the following formula

$\mathrm{CCD}_{\text {update }}$ can be derived as follows:

$$
\begin{aligned}
C C D_{\text {update }} & =f(S C D, E C D, V C D, R C D) \\
& =\alpha \times S C D+\beta \times E C D+\gamma \times V C D+\vartheta \times R C D .
\end{aligned}
$$

$\alpha, \beta, \gamma, \vartheta$ shall meet the following conditions: $0 \leq \alpha, \beta, \gamma, \vartheta \leq 1, \alpha+\beta+\gamma+\vartheta=1$.

Constrained clustering algorithm has made progress in solving scalability, but these features are not available in clustering algorithms in the field of statistics or machine learning. It is a hybrid clustering method based on grid and density, which better meets the above requirements. In a high-dimensional space, it can effectively cluster and find clusters nested in the subspace of high-dimensional data space. The basic idea of the algorithm is to combine the constraint condition with the antimonotone property of the constrained clustering algorithm and jointly use it to prune the candidate clusters, reduce the blindness in the searching process of the constrained clustering algorithm, and improve its efficiency and clustering quality. The general framework of the algorithm is shown in Figure 1.

Constraints, for instance, pairs, can be determined by parameters (Con $=$ : Must-Link constraint set, con $\neq$ : cannotlink constraint set). For example, in pair constraints, the basic idea of constraint clustering algorithm is as follows:

(i) Firstly, scan the dataset $D$ and cluster it on $K$ 1-dimensional spaces

(ii) Select dimension $D_{\min }$ with the smallest number of classes in $K 1$-spaces

(iii) The condition dense elements on $D_{\min }$ are obtained by imposing constraints on $D_{\min }$

(iv) Candidate dense cells are generated by using conditional dense cells on attribute $D_{\min }$ and other dense cells in $(k-1)$ 1-dimensional spaces

(v) The following steps are the same as those of the constrained clustering algorithm

3.2. Constraint Types and Algorithms. Domain knowledge is a very big concept, and there is almost no unified expression form. It can be expressed in various forms. In addition to the guidance form of "class label," there is information about the structure of the problem, heuristic guidance rules, the relationship between data objects, or the combination of the 


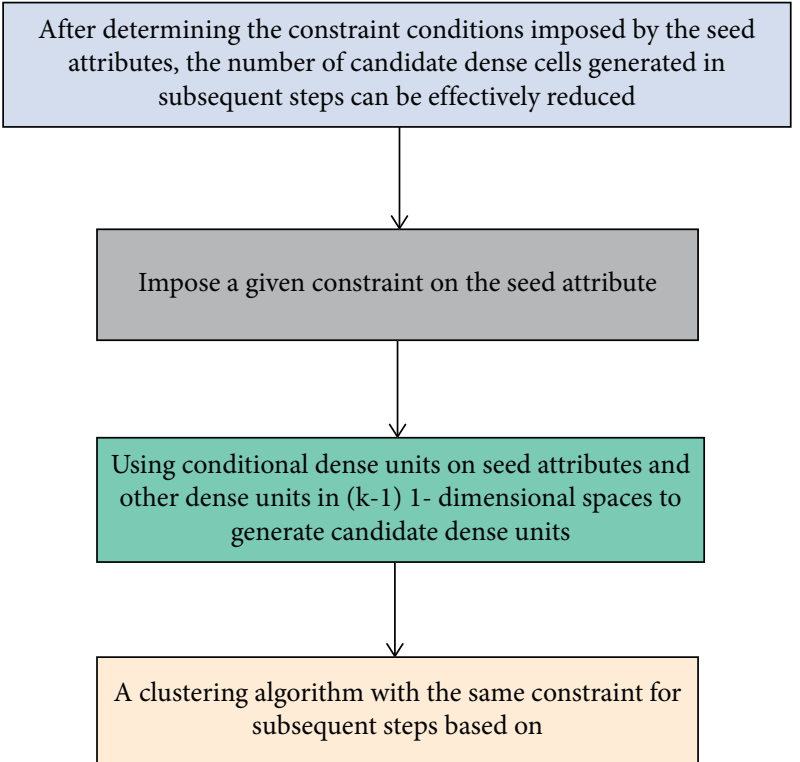

Figure 1: Overall framework of the constrained clustering algorithm.

above forms. The selection of central objects in the constrained clustering algorithm is random. If the randomly selected central objects are concentrated, the number of iterations to form the final partition may be increased, which affects the clustering speed. Therefore, selecting an appropriate central object can speed up the convergence of the algorithm. Since all spatial objects have spatial relationships, the appropriate central object can be selected according to the directional relationship of the residential object. According to the constraint clustering algorithm and matching algorithm, the association compactness of the pension industry chain is sorted from high to bottom. The ranking results of the association compactness of the pension industry chain are shown in Figure 2.

Then, combined with the enterprise reputation model, the optimized constrained clustering algorithm is used to calculate the association tightness of the elderly care industry chain. The numerical comparative analysis results before and after are shown in Figure 3.

Our algorithm is based on a mathematical morphology clustering algorithm. The obstacle constraint and the idea of dealing with the obstacle constraint are introduced. It not only has the advantages of a mathematical morphology clustering algorithm but also has low complexity. It can find clusters of arbitrary shapes. Moreover, the data on both sides of the obstacle can be effectively divided into different classes. The algorithm involves linear obstacles in two-dimensional space, and planar obstacles are considered as polygons surrounded by multiple linear obstacles. According to the scope of objects imposed by constraints, it can be divided into the following four categories:

(i) Global-level constraints work on the data objects participating in clustering.

(ii) Cluster-level constraints are constraints for each class, such as the number of data points owned in each class. (iii) Feature-level constraint is a constraint applied to the features describing data. When the features meet certain conditions, some rules are enabled.

(iv) Instance-level constraints are for data objects or constraints between data objects.

\section{Necessity of Transformation and Optimization of Pension Industry Chain}

4.1. Definition of Constrained Clustering. With the increasing aging of the population, however, the contradiction between the growing demand for the aged and the traditional family pension has become increasingly prominent due to the change of family structure and the acceleration of population mobility. In the traditional old-age care service industry, public old-age care institutions are supported by the government's financial support, forming a situation of long-term dependence on the government, which is often only targeted at specific subsidy groups. The institutional old-age care model can no longer meet the needs of the elderly, and the ecological old-age care model will become the development trend. The old-age care industry provides professional social services for the elderly at home to meet the diversified needs of the old-age care services. However, with the increase of the immunization rate, the reduction rate of the spread range has dropped all the way, especially when the proportion of the node enterprises implementing immunization exceeds 0.08 , the reduction rate of the spread range has obviously slowed down. As shown in the figure, the risk spread range caused by $h=08.0, h=1.0$, and $h=12.0$ has little difference. It can be seen that there is a threshold value for the proportion of immune enterprises when implementing the target immunization strategy for key node enterprises. In the aging population, accelerating family structure, and population mobility, the contradiction between the ever-increasing needs of providing for the aged and family support has become increasingly prominent. Social institutions for the aging population have been unable to meet the needs of providing for the elderly, and the way of providing for the aged population at home will become the future development trend. A municipal committee on aging released an analysis report chart of the aging situation of a certain population from 2015 to 2020. The latest data show that as of the end of 2019, the aging population in a certain city has increased the most. as shown in Figure 4.

According to the statistics in the table, the city has already entered an aging society, and the degree of aging is gradually deepening. The problem of population aging has profoundly impacted the social life, economic development, and cultural development of Wuhan. At the same time, it also puts forward tests and requirements for the social welfare and security system. Positive measures must be taken in the fields of pension medical care and pension insurance, and pension culture. In the process of sustainable social and economic development, transforming and optimizing industries can drive other industries in society. The pension industry intersects with many industries. For the special consumer groups of the elderly, the development of the pension industry can not only meet the pension needs of the elderly but also effectively stimulate domestic demand. The 


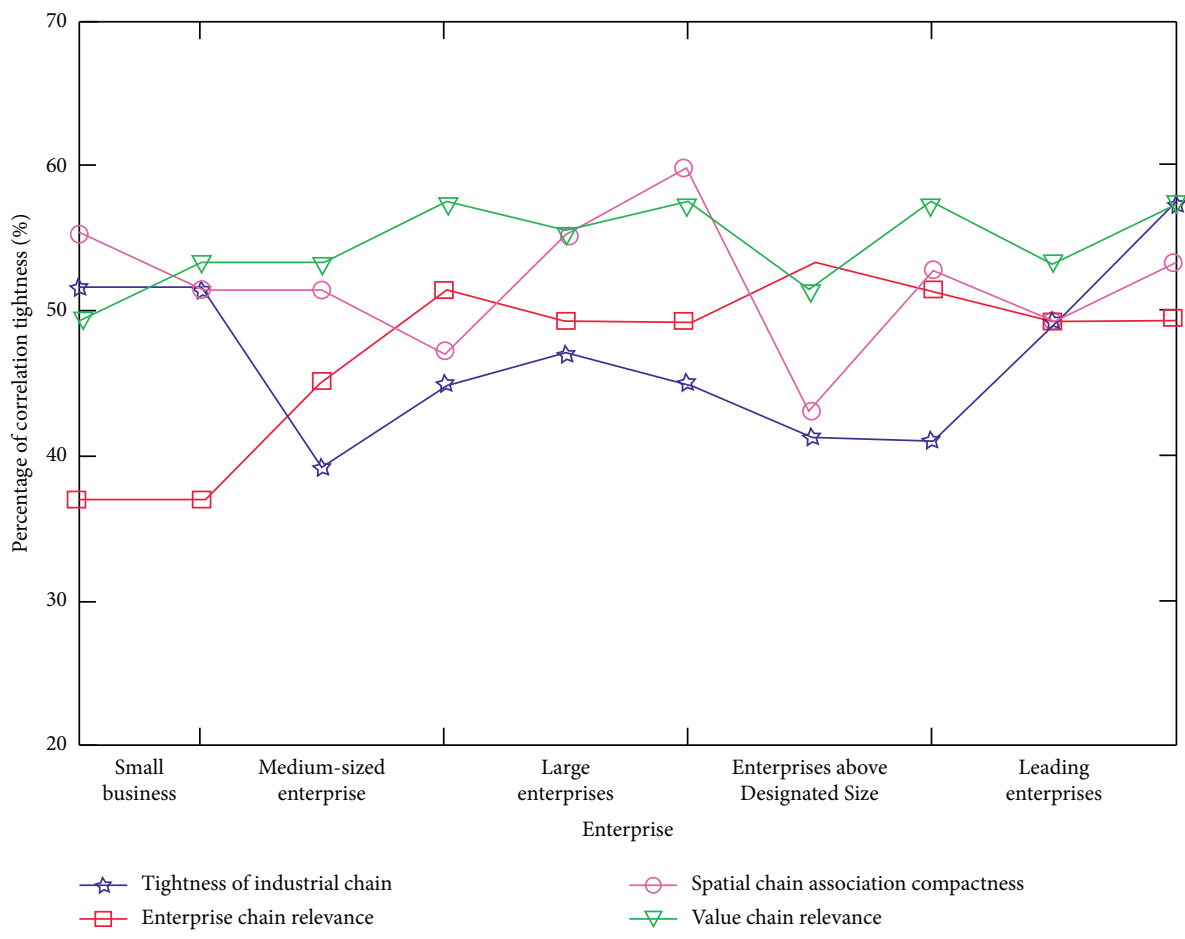

FIGURE 2: Ranking diagram of correlation degree of pension industry chain obtained by the constrained clustering algorithm.

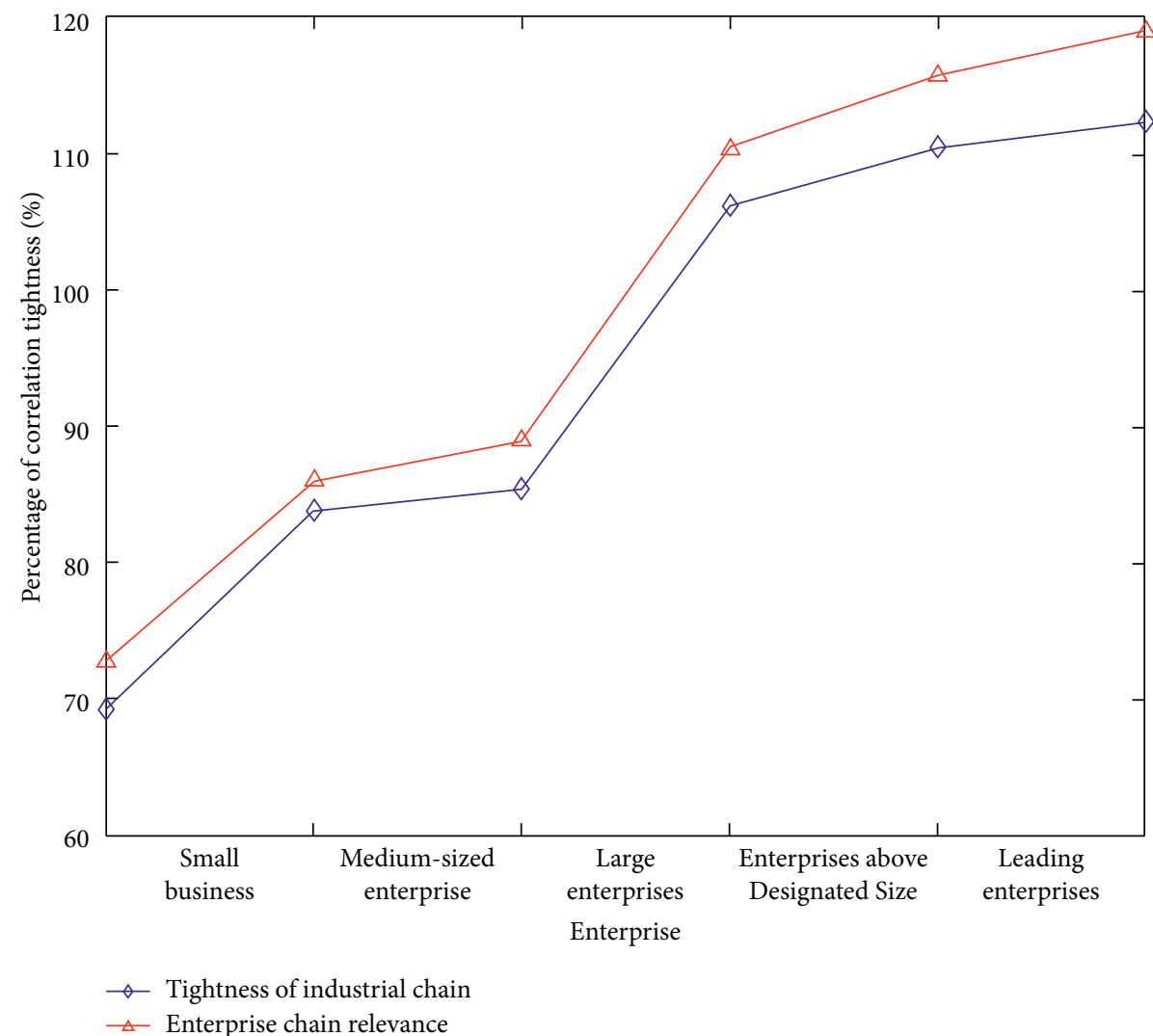

FiguRe 3: Comparison chart of correlation degree of pension industry chain before and after optimization.

change of risk propagation range of complex network of rural ecological elderly care industry chain based on target immunity and random immunity is shown in Figure 5.
It can be seen that the scope of risk spread has obviously shrunk after targeted immunization of industrial chain networks, with an average drop of more than $26 \%$. However, 


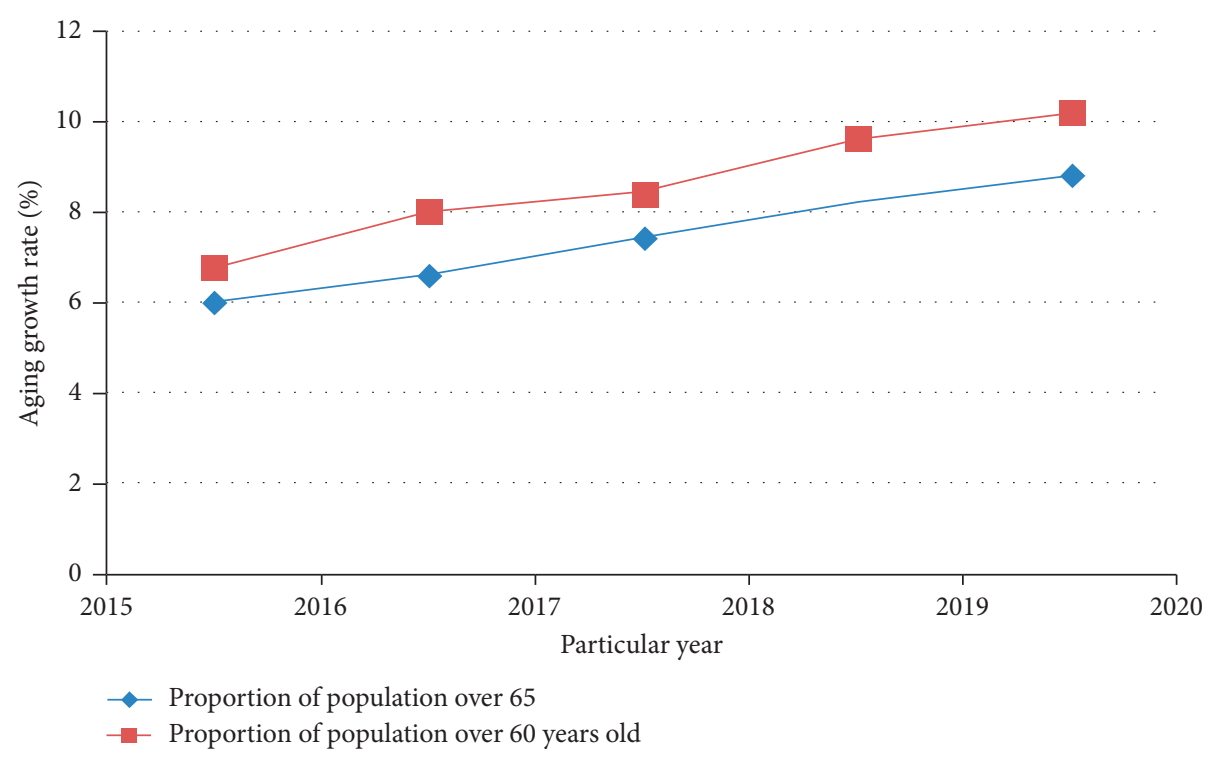

Figure 4: Population aging growth rate of a city.

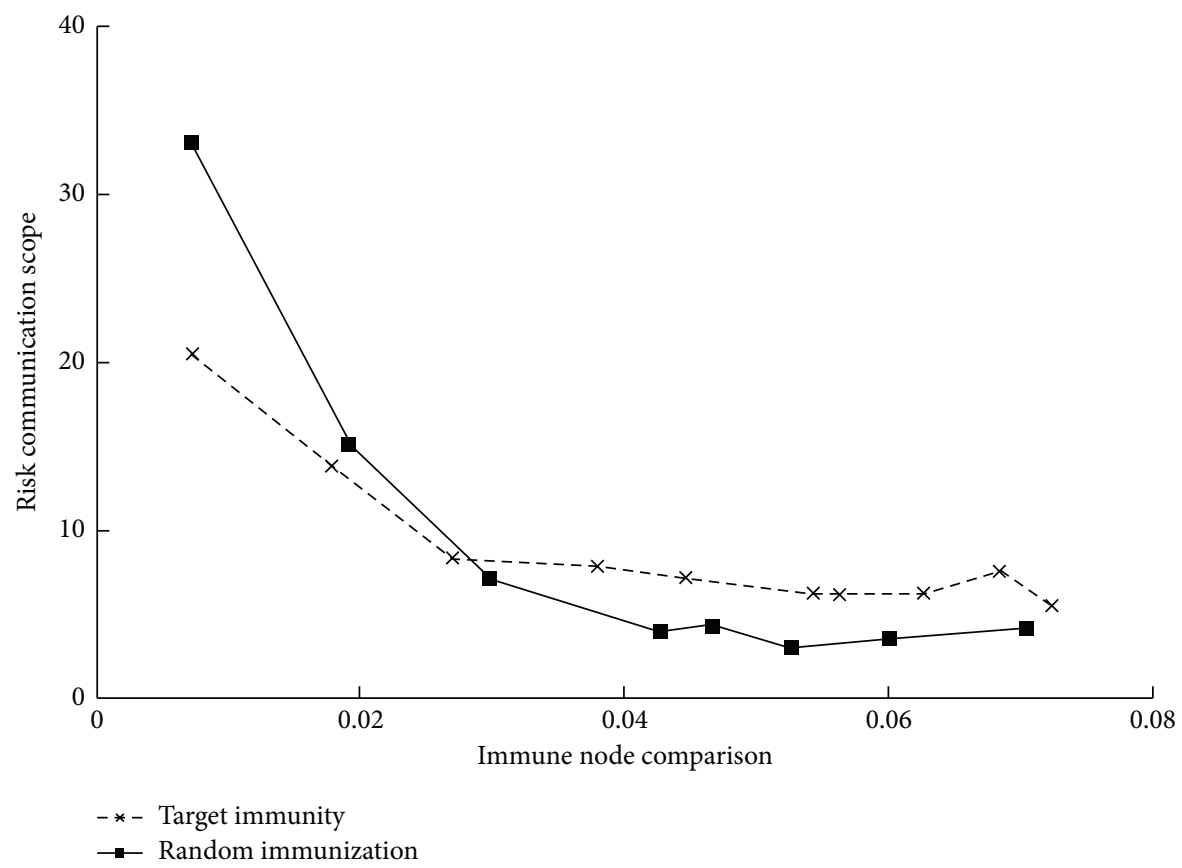

FIgURE 5: Change of risk transmission effect of pension industry chain under target immunization.

with the increase of the immunization rate, the reduction rate of the spread range has dropped all the way; especially when the proportion of the node enterprises implementing immunization exceeds 0.08 , the reduction rate of the spread range has obviously slowed down. As shown in the figure, the risk spread range caused by $h=08.0, h=1.0$, and $h=12.0$ has little difference. It can be seen that there is a threshold value for the proportion of immune enterprises when implementing the target immunization strategy for key node enterprises. When the proportion of immune enterprises is less than the threshold value, the control effect of industrial chain risk transmission is very obvious with the increase of the proportion. When the proportion of immune enterprises is greater than the threshold value, increasing the immune enterprises does not cause a significant reduction in the scope of risk transmission.

Private old-age care institutions built by social forces are difficult to maintain and operate, with few sources of funds, low profits, and no good industrialization form. The transformation and optimization of the old-age care industry should be accelerated, and high-quality life products and services for the elderly should be better provided. Comparatively speaking, the investment risk of social capital is relatively high, and a complete mature model of 
investment in old-age care institutions has not yet been formed, and most of the capital is still on the sidelines, which makes it difficult for the government to really implement measures to encourage social forces to enter the old-age care industry.

\subsection{The Need to Promote Sustainable Socioeconomic} Development. The transformation and optimization of the old-age care industry are critical engines for future economic growth, with a significant economic driving effect. The oldage care industry is a sunrise industry with many industries, and the old-age care industry's market supply system, which is generated by the satisfaction of six "old-age" needs, has a lot of room for growth. The old-age care industry has its own unique characteristics, and its core components are old-age care services and old-age care products, and the old-age care industry chain is comprised of many aspects of economy and society, a long industrial chain, reliance and interaction with the environment, and requirements for sustainable development. In a market economy, vigorously developing the pension industry is an unavoidable requirement for the development of population aging. The retirement industry will usher in a new era of rapid growth. As an emerging industry, The pension industry is critical to adjusting the industrial structure, improving employment rates, promoting coordinated economic and social development, and improving the socialist market economy. The chain of ecological pensions is usually in a state of rapid development. Although the market has recognized new products to some extent, more capital investment and technological advancement are still required to expand market share. Figure 6 depicts the parameters.

Let $U=\left[\mu_{i k}\right]_{c \times n}$ be a hard partition matrix, where $C$ is the number of clusters, which is the number of samples. The cluster center point set of a partition $P=\left\{P_{1}, P_{2}, \ldots, P_{C}\right\}$. According to the sample division method, then

$$
\begin{aligned}
\mu_{i k} & = \begin{cases}1, & d_{p i k}=\min \left\{d_{p 1 k}, d_{p 2 k}, \ldots, d_{p c k}\right\} \\
0, & \text { Other },\end{cases} \\
k & =1,2, \ldots, n ; \quad i=1,2, \ldots, c
\end{aligned}
$$

where $d_{P i k}$ is the reachable distance between the sample $X_{k}$ in class I and the center point pi of class I. If the sum of withinclass squared errors (WGSE) is used as the clustering objective function, then the clustering objective function is as follows:

$$
f(U, P)=\sum_{k=1}^{n} \sum_{i=1}^{c} \mu_{i k}\left(d_{p i k}\right)^{2} .
$$

If the natural coding scheme is adopted, the volume coding is

$$
b=\left\{\operatorname{Num}_{p 1}, \operatorname{Num}_{p 2}, \ldots, \operatorname{Num}_{p}, \ldots, \operatorname{Num}_{p c}\right\},
$$

where $\operatorname{Num}_{p i}\left(1 \leq \operatorname{Num}_{p i} \leq n\right)$ means that the cluster center $p_{i}(i=1,2, \ldots, c)$ is taken from the $\mathrm{Num}_{p i}$ sample in the sample set. According to our knowledge of analytic geometry, the edges $\left(x_{i}, x_{j}\right)$ and edges $\left(y_{k m}, y_{k}(m+1)\right)$ meet the following conditions:

$\left\{\begin{array}{l}\left(a_{l} \times y_{k m l}+b_{l k m 2}+c_{1}\right) \times\left(\alpha_{1} \times y_{k(m+1) l}+b_{l y k(m+1) 2}+C_{1}\right)<0, \\ \left(a_{2} \times x_{i 1}+b_{2} x_{i 2}+c_{2}\right) \times\left(\alpha_{2} \times x_{j 1}+b_{2} x_{j 2}+c 2\right)<0 .\end{array}\right.$

Based on the matching model of the same evaluation index, the following mathematical model is established:

$$
\begin{gathered}
\max \sum_{i=1}^{I} \sum_{j=1}^{J} \alpha_{i j} * x_{i j}+\sum_{i=1}^{I} \sum_{j=1}^{J} \beta_{i j} * x_{i j}, \\
0<\sum_{j=1}^{I} x_{i j} \leq p_{i}, \forall_{i}, j,
\end{gathered}
$$

$$
0<\sum_{i=1}^{I} x i_{j} \leq q_{j}, \forall i, j .
$$

In the above model, (8) is the objective function, which means to maximize the total matching degree of matching parties as much as possible. Formula (9) means that the caregiver I matches pi loved ones at most. Formula (10) means that the cared-for $j$ matches $q_{j}$ caregivers at most. Moreover, (4) is a $0-1$ decision variable.

Compared with the traditional elderly care industry, the ecological elderly care industry is a new industry that gives full play to the ecological advantages of resources and environment so as to meet the needs of elderly care and leisure services. This kind of industry has the characteristics of low-carbon and environmental protection. Through appropriate government guidance and policy support, the development of the pension industry is bound to inject new vitality into China's current economic development, promote new growth of the national economy, and further promote the sustainable development of the current economy and society.

4.3. The Need to Improve the Level and Efficiency of the Aged Care Service Industry. The development of China's pension industry lags behind, the supply of pension products and services is insufficient, and the pension industry chain is immature. Therefore, the transformation and optimization of the pension industry are imperative. It can be seen that the scope of risk spread has obviously shrunk after targeted immunization of industrial chain networks, with an average drop of more than $26 \%$. In addition, the elderly care service is inseparable from the care and greetings of the elderly. When the proportion of immune enterprises is less than the 


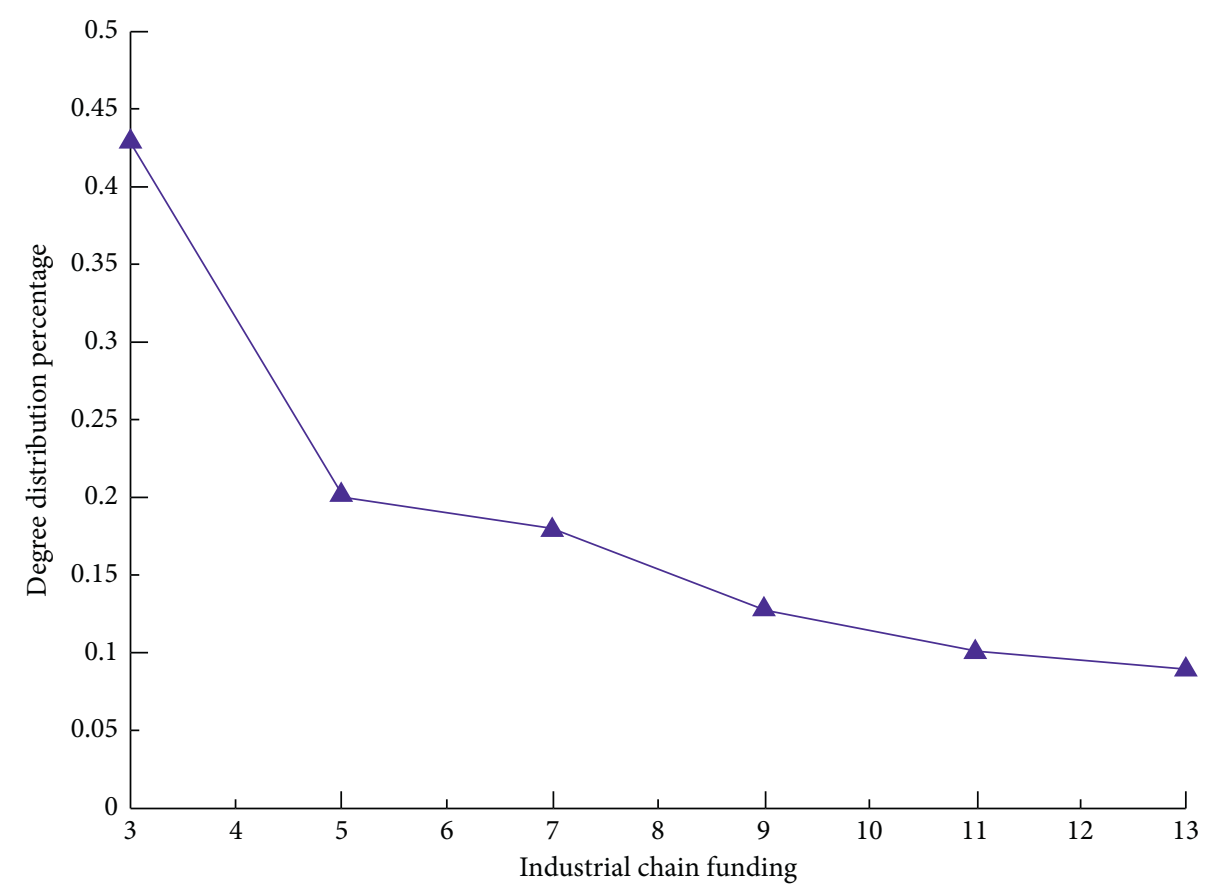

FIGURE 6: Distribution chart of capital change degree of the ecological pension industry chain.

threshold value, the control effect of industrial chain risk transmission is very obvious with the increase of the proportion. In the care greeting network, both the caring person and the cared person contain multiple matching indicators. Under the centralized construction mode of care greeting network, matching is carried out according to the indicators of the caring person and the cared person, so as to achieve the overall optimal goal of the network. In the network of caring and greeting, the main consideration is the matching between the caring person and the cared person. There are both the same matching indicators and different matching indicators between them, as shown in Figure 7 .

Matching indicators of loved ones are educational background, work experience, geographical location, and service demand. Caregiver matching indicators are educational background, work experience, geographical location, knowledge, and skills. In the process of matching, each matching index is matched in one-to-one correspondence, in which the service needs of the loved ones are matched with the knowledge and skills of the loved ones.

According to the educational background, match the schools of the caregivers and the loved ones, take the names of different schools as the matching basis, and calculate the number of schools where the two sides have the same attendance so as to determine the matching degree. In the care and greeting network, caregivers and loved ones continue to join through the network platform, and we are concerned about how to reasonably match them. In the matching process, taking the optimal overall matching degree as the goal, under multiple matching indexes, the matching degree between matching objects is calculated according to the individual preference of matching objects, and then the connection between them is established intensively. In the care and greeting network, it is assumed that there are six caregivers and six loved ones. The maximum matching number of caregivers is 3 and the maximum matching number of loved ones is 6 . The matching degree between caregivers and loved ones is shown in Figure 8.

From the perspective of the industrial chain, such distribution characteristics reflect the following. (1) There is no significant difference in the number of partners between node enterprises, which means that although a small number of core enterprises with comparative advantages have been formed in the industrial chain, this advantage is not obvious. (2) There are a large number of enterprises with a medium competitive advantage, and some of them may stand out and become industry leaders. (3) The number of enterprises in the third echelon is small, and there are mainly two types. First, enterprises or institutions with special properties, such as product certification bodies, mainly provide certification services for a small number of enterprises, so there are fewer partners in a single industrial chain. Second, the backward enterprises in the industrial chain, whose development scale is small and the speed is slow, cannot win a large number of partners and are likely to be eliminated in the competition, as shown in Figure 9.

By sorting out the factors that affect the industry consolidation for the aged population, this article sets six potential variables that can not be directly observed, namely, the environment of the aged care service, the demand structure of the aged group, the management of the aged care service, the intelligent aged care service system, the development status of the intelligent aged care industry, and the service feedback effect. Each potential variable is represented by a group of significant observable variables, with a total of 16 significant variables. The theoretical framework of 


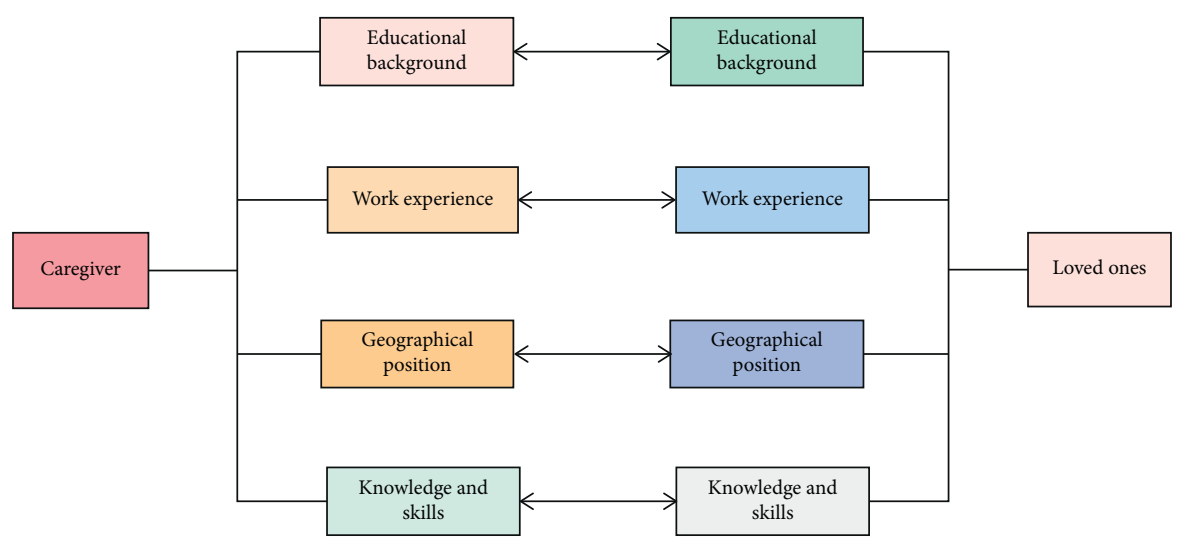

FIgURE 7: Matching relationship of network indicators.

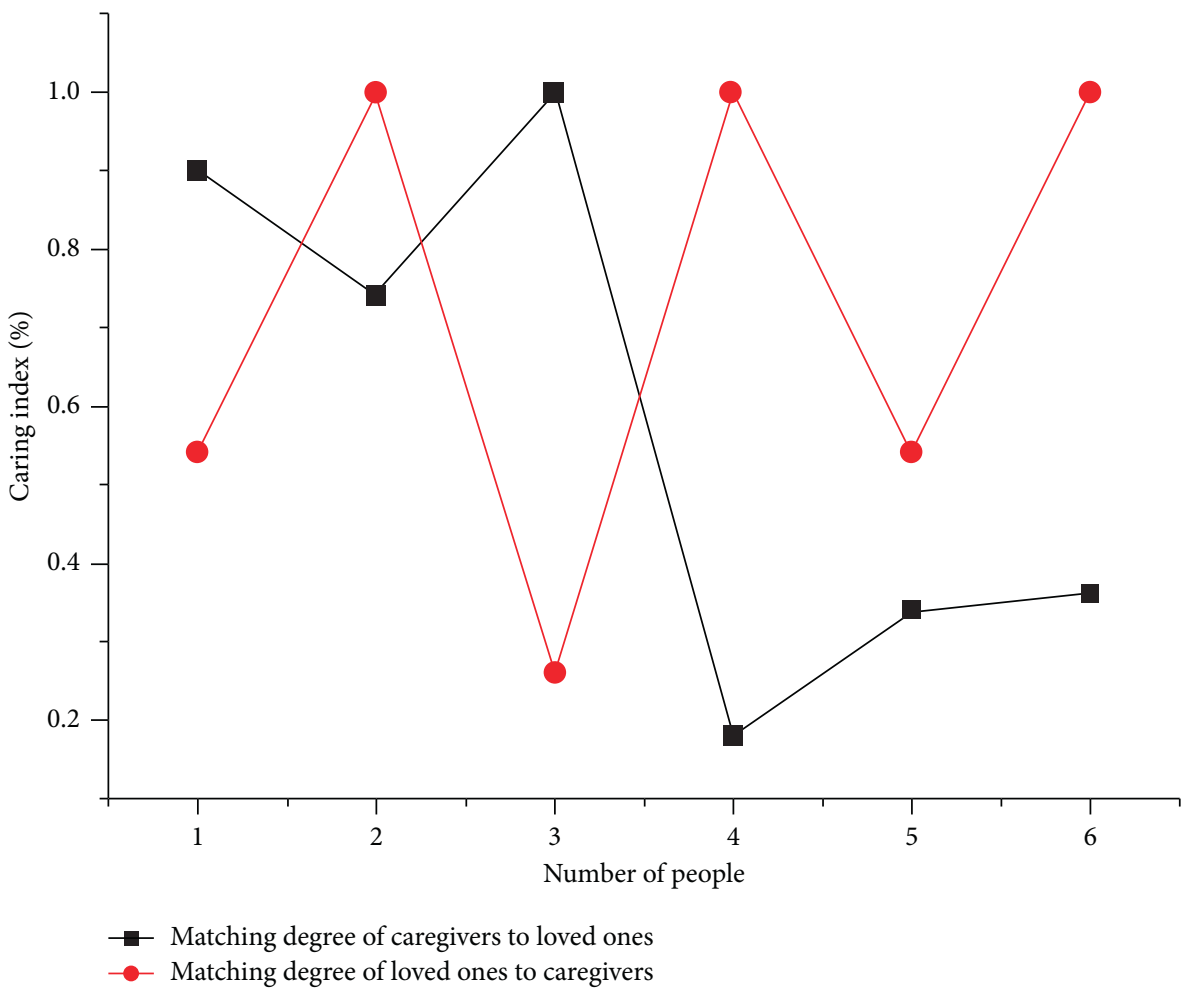

FIgURE 8: The matching degree between the caregiver and the loved one.

"triple functional relationship" mainly refers to the analysis framework of the influencing factors of the industry consolidation of smart pension, as shown in Figure 10.

With the increasingly prominent problem of population aging, it is necessary to promote the modernization, scale, industrialization, specialization, and institutionalization transformation of traditional aged care services, from the government to the combination of government-led and market-oriented mechanisms and from the traditional lowlevel aged care services to the high-level aged care services.
Farmers should be encouraged to implement land circulation and form agricultural cooperatives through various forms such as joint ventures and shareholding so as to make full use of local ecological advantages and make use of idle land. The transformation and optimization of the old-age care industry should be accelerated, and high-quality life products and services for the elderly should be better provided. It is important to carry out investment attraction, establish a special management committee, reasonably allocate and coordinate relevant resources, and carry out 


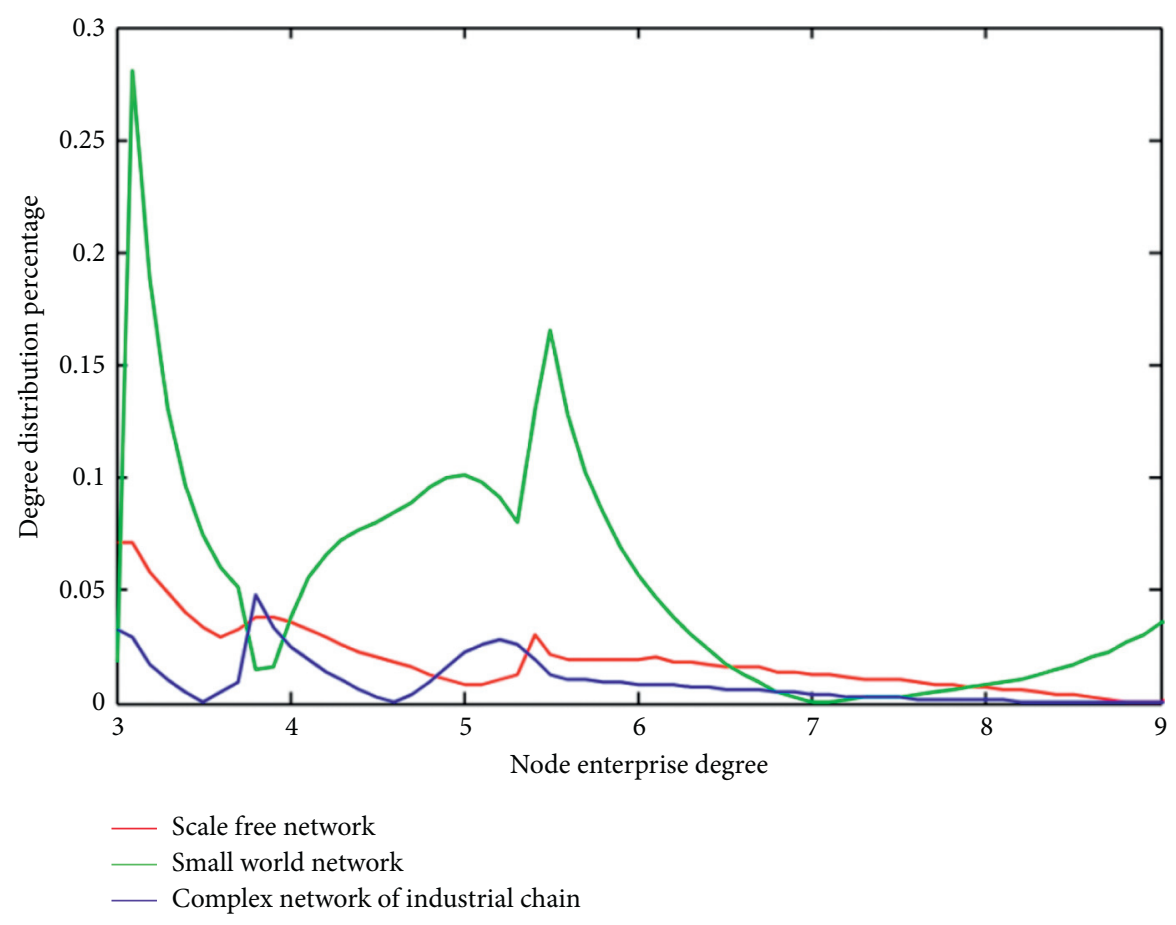

Figure 9: Degree distribution of three network structures.

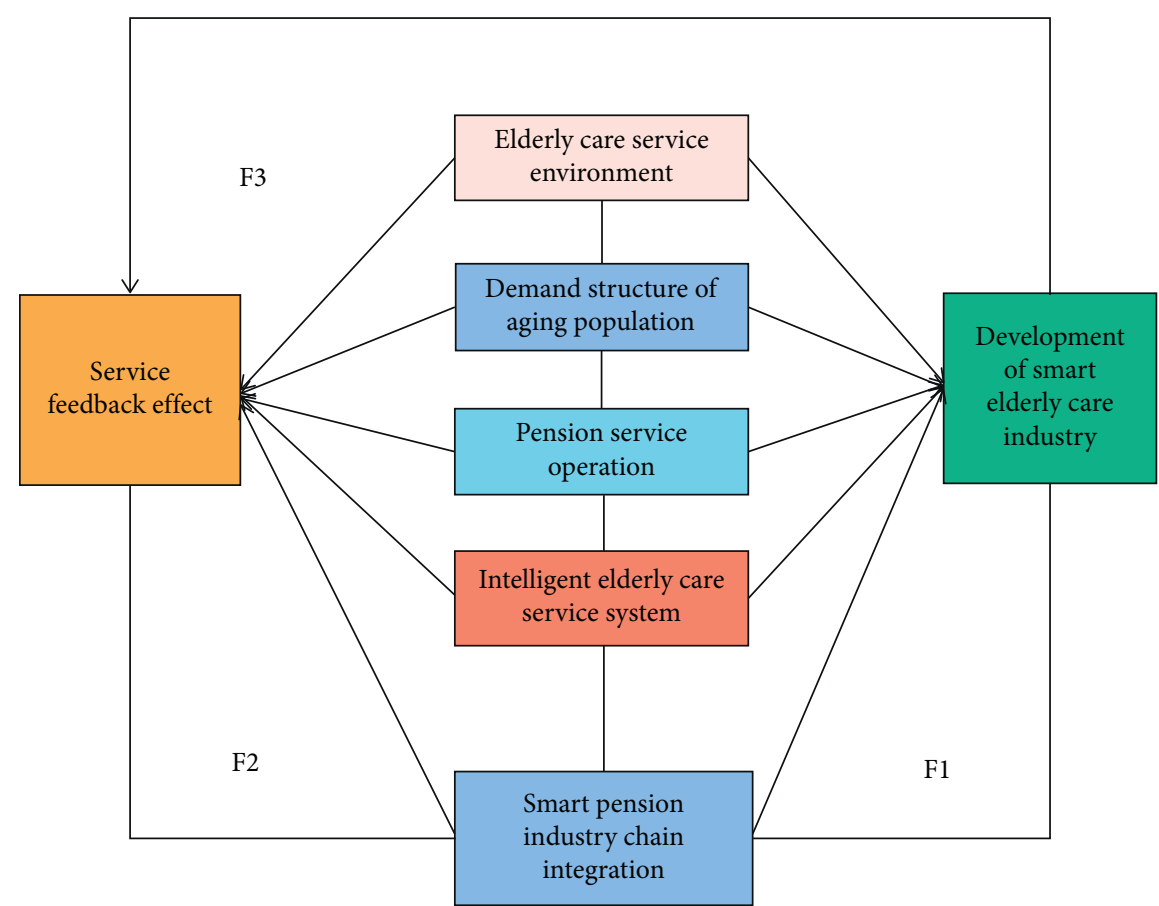

FIGURE 10: Theoretical framework of "triple function relationship" of influencing factors of pension industry chain integration.

classified management. Farmers can operate some small farmhouses, and enterprises can invest in large sightseeing parks.

\section{Conclusions}

In a word, in the process of increasing the social aging population, strengthening the transformation and development of elderly products is of great significance to the safe and stable development of the whole society and effectively promotes the development of the social economy. This article studies the effectiveness and importance of constrained clustering algorithms in the transformation and optimization of rural ecological elderly care industrial chains. The choice of ecological pension industry model is one of the frontier issues in the economic theory research of 
the pension industry, and the research on the implementation path of rural revitalization is the main focus of the development theory research of "agriculture, rural areas, and farmers." Private old-age care institutions built by social forces are difficult to maintain and operate, with few sources of funds, low profits and no good industrialization form. At present, the academic circles have made some progress in researching the mode selection of the ecological pension industry. Although scholars have different opinions on the specific mode selection, they have reached a consensus in some aspects. As the ecological pension industry is a new industrial form in China, and the rural revitalization strategy has only been put forward in recent years and the research on it by domestic scholars is extremely limited and is still in the primary exploration stage. The effective use of background knowledge can make the constrained clustering algorithm obtain more heuristic information to reduce the blindness in the search process and improve the efficiency and clustering quality of the algorithm. This article describes in detail the role and advantages of the constrained clustering algorithm in transforming the rural ecological elderly care industrial chain. At the same time, we should also strengthen the supervision of various forms of community pension models such as private and public assistance, public construction, and private and public construction. In the process of supervising these forms of old-age care, we also need to pay attention to matters such as staffing, investment and use of government funds, and service quality.

\section{Data Availability}

The data used to support the findings of this study are included within the article.

\section{Conflicts of Interest}

The authors declare that they have no conflicts of interest.

\section{References}

[1] W. Li, L. Wang, X. Cai, and J. Hu, "Species co-evolutionary algorithm: a novel evolutionary algorithm based on the ecology and environments for optimization," Neural Computing and Applications, vol. 31, no. 7, pp. 2015-2024, 2019.

[2] R. Jiao, S. Zeng, C. Li, S. Yang, and Y. -S. Ong, "Handling constrained many-objective optimization problems via problem transformation," IEEE Transactions on Cybernetics, vol. 51, no. 99, pp. 1-14, 2020.

[3] C. W. Hu, H. Li, and A. A. Qutub, "Shrinkage Clustering: a fast and size-constrained clustering algorithm for biomedical applications," BMC Bioinformatics, vol. 19, no. 1, p. 19, 2018.

[4] M. R. Sharifi, S. Akbarifard, K. Qaderi, and M. R. Madadi, "Comparative analysis of some evolutionary-based models in optimization of dam reservoirs operation," Scientific Reports, vol. 11, no. 1, Article ID 15611, 2021.

[5] N. Liu, Z. Xu, Y. He, and X. -J. Zeng, "An inverse prospect theory-based algorithm in extended incomplete additive probabilistic linguistic preference relation environment and its application in financial products selection," Fuzzy Optimization and Decision Making, vol. 20, 2021.
[6] A. Shabani, B. Asgarian, M. A. Salido, and S. Asil Gharebaghi, "Search and Rescue Optimization Algorithm: A New Optimization Method for Solving Constrained Engineering Optimization problems," Expert Systems with Applications, vol. 161, Article ID 113698, 2020.

[7] A. S. Eesa and Z. Orman, "A new clustering method based on the bio-inspired cuttlefish optimization algorithm," Journal of Expert Systems, vol. 37, 2020.

[8] G. Dhiman and V. Kumar, "Seagull optimization algorithm: theory and its applications for large-scale industrial engineering problems," Knowledge-Based Systems, vol. 165, no. 1, pp. 169-196, 2019.

[9] R. Liu, W. Cai, G. Li, X. Ning, and Y. Jiang, "Hybrid dilated convolution guided feature filtering and enhancement strategy for hyperspectral image classification," IEEE Geoscience and Remote Sensing Letters, vol. 10, 2021.

[10] Y. Y. Zheng, J. L. Kong, X. B. Jin, X. Y. Wang, T. L. Su, and M. Zuo, "CropDeep: the crop vision dataset for deep-learning-based classification and detection in precision agriculture," Sensors, vol. 19, no. 5, p. 1058, 2019.

[11] W. Cai, D. Liu, X. Ning, C. Wang, and G. Xie, "Voxel-based three-view hybrid parallel network for 3D object classification," Displays, vol. 69, Article ID 102076, 2021.

[12] J. Kong, H. Wang, X. Wang, X. Jin, X. Fang, and S. Lin, "Multi-stream hybrid architecture based on cross-level fusion strategy for fine-grained crop species recognition in precision agriculture," Computers and Electronics in Agriculture, vol. 185, Article ID 106134, 2021.

[13] F. Mach, "Reduction of optimization problem by combination of optimization algorithm and sensitivity analysis," IEEE Transactions on Magnetics, vol. 52, no. 3, pp. 1-4, 2016.

[14] F. Ros and S. Guillaume, "DIDES: a fast and effective sampling for clustering algorithm," Knowledge and Information Systems, vol. 50, no. 2, pp. 543-568, 2017.

[15] M. Anusha and J. Sathiaseelan, "Evolutionary clustering algorithm using criterion-knowledge-ranking for multi-objective optimization," Wireless Personal Communications, vol. 94, no. 4, pp. 1-22, 2016.

[16] H. Zhang and Y. Wu, "Optimization and Application of Clustering Algorithm in Community Discovery," Wireless Personal Communications, vol. 102, 2018.

[17] M. Zhao, J. Aadarsh, L. Quan et al., "Faster Mean-shift: GPUaccelerated clustering for cosine embedding-based cell segmentation and tracking," Medical Image Analysis, vol. 71, Article ID 102048, 2021.

[18] $\mathrm{Z}$. Wu and W. Chu, "Sampling strategy analysis of machine learning models for energy consumption prediction," in Proceedings of the 2021 IEEE 9th International Conference on Smart Energy Grid Engineering (SEGE), August 2021.

[19] Z. Huang, Y. Zhang, Q. Li et al., "Unidirectional variation and deep CNN denoiser priors for simultaneously destriping and denoising optical remote sensing images," International Journal of Remote Sensing, vol. 40, no. 15, pp. 5737-5748, 2019.

[20] L. Zhang and M. Jin, "A constrained clustering-based blind detector for spatial modulation," IEEE Communications Letters, vol. 23, no. 7, pp. 1170-1173, 2019.

[21] H. Sun, F. Zhu, Y. Hao et al., "Unified optimization for multiple active object recognition tasks with feature decision tree," Journal of Intelligent and Robotic Systems, vol. 103, no. 2, pp. 1-15, 2021.

[22] L. Abualigah and A. Diabat, "A Comprehensive Survey of the Grasshopper Optimization Algorithm: Results, Variants, and applications," Neural Computing and Applications, vol. 12, 2020. 
[23] Q. Fang, H. Nguyen, X. N. Bui, T. Nguyen-Thoi, and J. Zhou, "Modeling of rock fragmentation by firefly optimization algorithm and boosted generalized additive model," Neural Computing and Applications, vol. 33, no. 2, 2020.

[24] M. Khishe and M. R. Mosavi, "Classification of underwater acoustical dataset using neural network trained by Chimp Optimization Algorithm," Applied Acoustics, vol. 157, Article ID 107005, 2020.

[25] K. Li, J. Luo, Y. Hu, and S. Li, "A novel multi-strategy DE algorithm for parameter optimization in support vector machine," Journal of Intelligent Information Systems, vol. 54, no. 3, pp. 527-543, 2020.

[26] J. Zhou, C. Gao, W. Pedrycz, L. Zhihui, and Y. Xiaodong, "Constrained shadowed sets and fast optimization algorithm," International Journal of Intelligent Systems, vol. 34, no. 6, 2019.

[27] K. Zanbouri and N. J. Navimipour, "A cloud service composition method using a trust-based clustering algorithm and honeybee mating optimization algorithm," International Journal of Communication Systems, vol. 33, no. 8, 2019.

[28] Z. Cao and L. Wang, "A Comprehensive Study of Phase Based Optimization Algorithm on Global Optimization Problems and its applications," Applied Intelligence, vol. 49, 2019.

[29] T. Min, W. Fang-Xiang, P. Yi, and W. Jianxin, "SCOP: A Novel Scaffolding Algorithm Based on Contig Classification and Optimization," Bioinformatics, vol. 35, 2018.

[30] O. R. Castro, A. Pozo, J. A. Lozano, and S. Roberto, "An investigation of clustering strategies in many-objective optimization: the I-Multi algorithm as a case study," Swarm Intelligence, vol. 11, no. 2, pp. 101-130, 2017.

[31] K. H. Redford, Ecology and power in the age of empire: europe and the transformation of the tropical world by corey ross, Oxford University Press, vol. 53, no. 02, Oxford, UK, 2017. 